\title{
Hard QCD \\ and Hadronic Final State at HERA
}

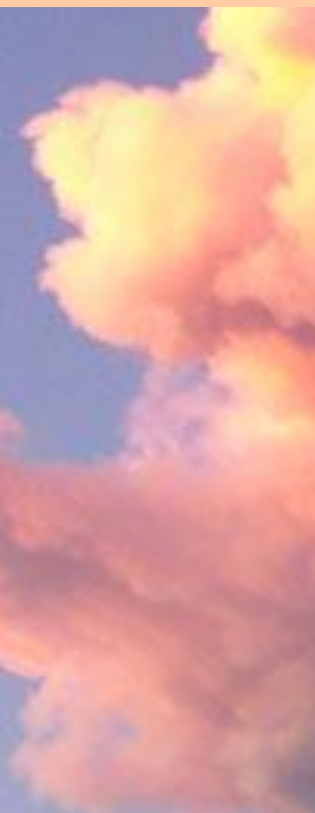
Alice Valkárová, on behalf of $\mathrm{H} 1$ and ZEUS
Collaborations

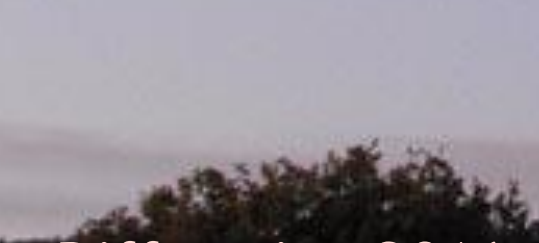




\section{HERA collider experiments}

- $27.5 \mathrm{GeV}$ electrons/positrons on $920 \mathrm{GeV}$ protons $\rightarrow \sqrt{ } \mathrm{s}=318 \mathrm{GeV}$

- data taken in 1992-2007

- HERA I,II: $500 \mathrm{pb}^{-1}$ per experiment

- H 1 \& ZEUS - 4T detectors
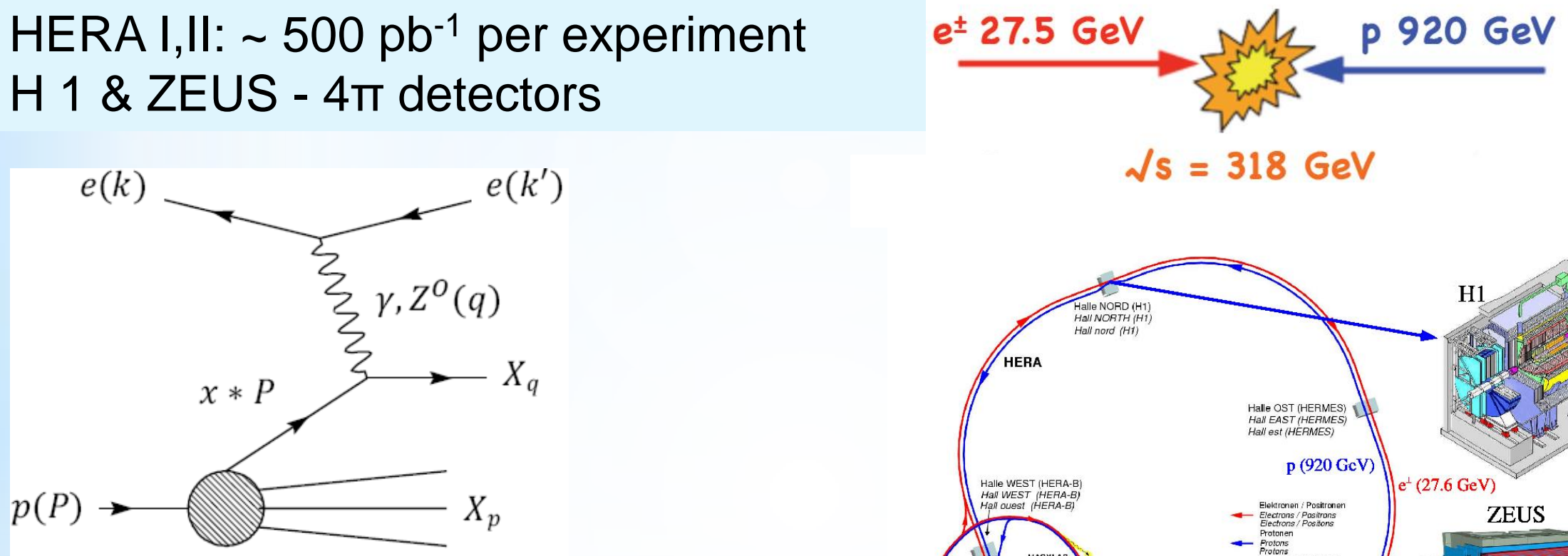

$$
\sqrt{s}=318 \mathrm{GeV}
$$

Virtuality of exchanged boson $Q^{2}=-q^{2}=-\left(k-k^{\prime}\right)^{2}$

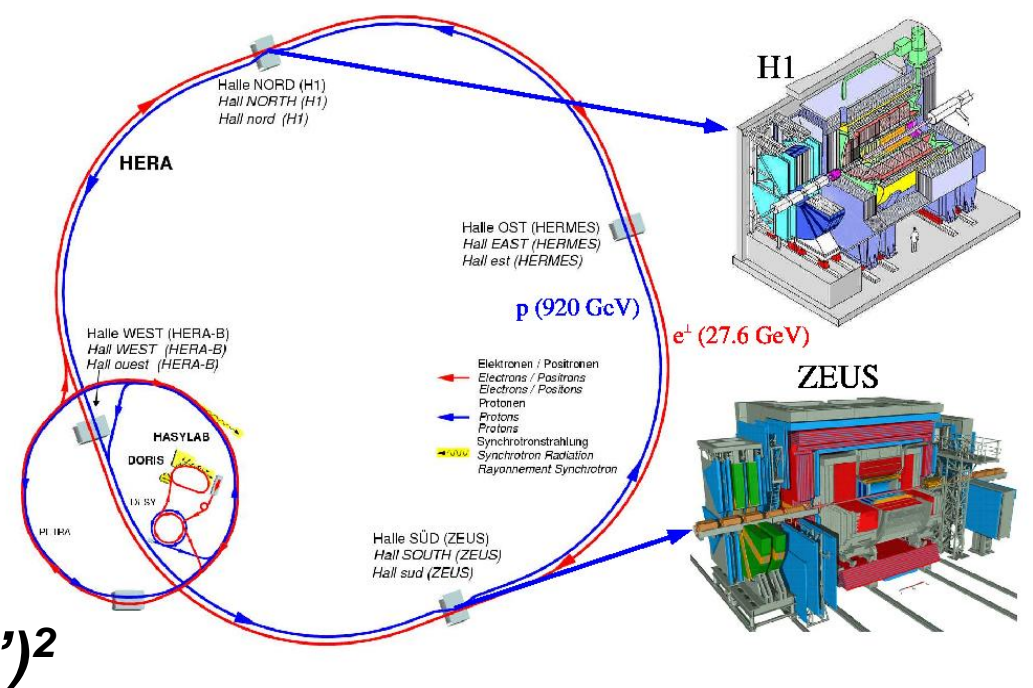

Inelasticity $\boldsymbol{y}=\boldsymbol{P q} / \boldsymbol{P k}$

Bjorken scaling variable $x=Q^{2} / 2 q P$
Two regimes:

$\mathrm{Q}^{2}<1 \mathrm{GeV}^{2}$ photoproduction ( $\left.r p\right)$

$Q^{2} \gg 1 \mathrm{GeV}^{2}$ Deep Inelastic Scattering (DIS) 


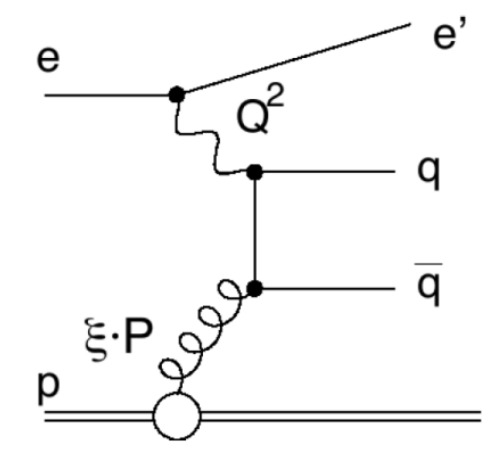

Boson-gluon fusion

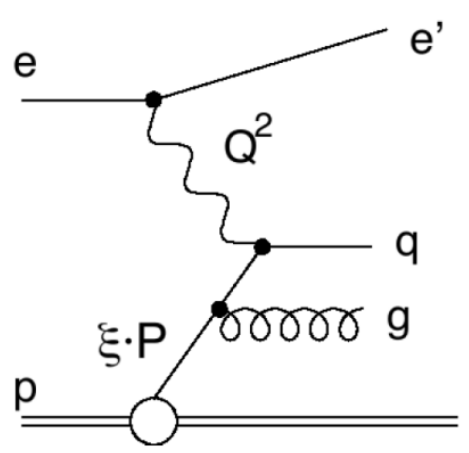

QCD Compton

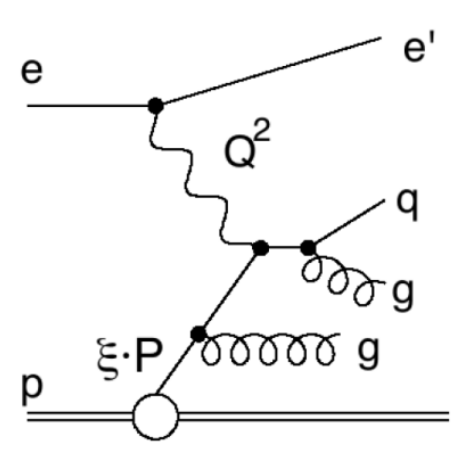

Trijet leading-order
Jet measurements in Breit reference frame $\rightarrow$

exchanged virtual boson collides head on with parton from proton

Dijet measurement: boson-gluon fusion \& QCD Compton sensitive to $O\left(\alpha_{s}\right)$ already at LO

Trijet measurement: calculations in PQCD in LO already at $O\left(\alpha_{s}{ }^{2}\right)$

Process

inclusive

Low $Q^{2}$ dijets

trijets

inclusive

High $Q^{2}$ dijets

$Q^{2}>150 \mathrm{GeV}^{2}$ trijets
H1

HERA I

EPJC 67

(2010) 1

EPJC 65

(2010) 363

\section{H1}

HERAII

This analysis

H1prelim 16-061

H1prelim 16-062

EPJC 75 (2015) 2

This analysis

H1prelim 16-062
ZEUS

HERA I

HERA II

Nucl.Ph.B 765

(2007) 1

PL B 691

EPJC 70

(2010) 127 
Data: HERA II period 2006-2007 Integrated luminosity $L=184 \mathrm{pb}^{-1}$ Regularised unfolding procedure S.Schmitt,JINST 7(2012)T10003 Takes into account kinematic migration by considering an 'extended phase space' $\rightarrow$ describes accurately migrations into and out of final 'measurement phase space'

H1 prelim-16-061,H1 prelim-16-062 included also high $\mathrm{Q}^{2}$ data from Eur.Phys.J.C75 (2015) 2, 65

- Inclusive $\mathrm{k}_{\mathrm{t}}$ algorithm with $\mathrm{R}=1$

- Jet energy calibration using neural networks

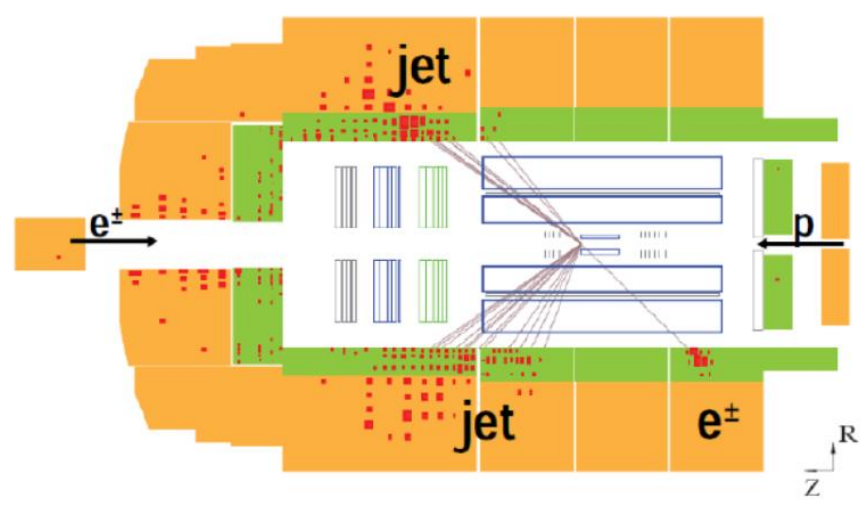

$$
-1.5<\eta_{\mathrm{lab}}^{\mathrm{jet}}<2.5
$$

Phase space low $Q^{2} \quad$ Phase space high $Q^{2}$

NC DIS phase space

$5.5<\mathrm{Q}^{2}<80 \mathrm{GeV}^{2}$

$0.2<y<0.6$

$150<Q^{2}<15000 \mathrm{GeV}^{2}$

$0.2<y<0.7$

$4.5<\mathrm{P}_{\top}{ }^{\text {jet }}<50 \mathrm{GeV}$

$5<\mathrm{p}_{\mathrm{T}}^{\text {jet }}<50 \mathrm{GeV}$

$5<<\mathrm{p}_{\mathrm{T}}>_{2}<50 \mathrm{GeV}$

Dijets Njet $\geq 2$

Trijets Njet $\geq 3$
$5.5<<\mathrm{p}_{\mathrm{T}}>_{3}<40 \mathrm{GeV}$ 


\section{MC models and NLO QCD}

\section{Monte Carlo generators:}

RAPGAP: LO matrix elements +PS

DJANGOH:Color-dipole model -Ariadne Lund string fragmentation for hadronisation

\section{NLO calculations:}

- nlojet++ (Z.Nagy et al.), 5 quarks

- with NNPDF 3.0

(includes full H1\&ZEUS HERAIl DIS data)

$-\alpha_{s}=0.118$ (as in PDF)

- renormalisation and factorisation scales: $\mu_{r}=\mu_{f}=\sqrt{\left(\left(P_{T^{2}}+Q^{2}\right) / 2\right)}$

\section{aNNLO and NNLO calculations:}

Normalised with NC DIS NNLO

predictions (APFEL)

- aNNLO - JetViP, approximate NNLO using threshold resummation, PR D 92 (2015) 074037

- NNLO - NNLOJET

J.Currie at al, PRL, 117 (2016) 042001
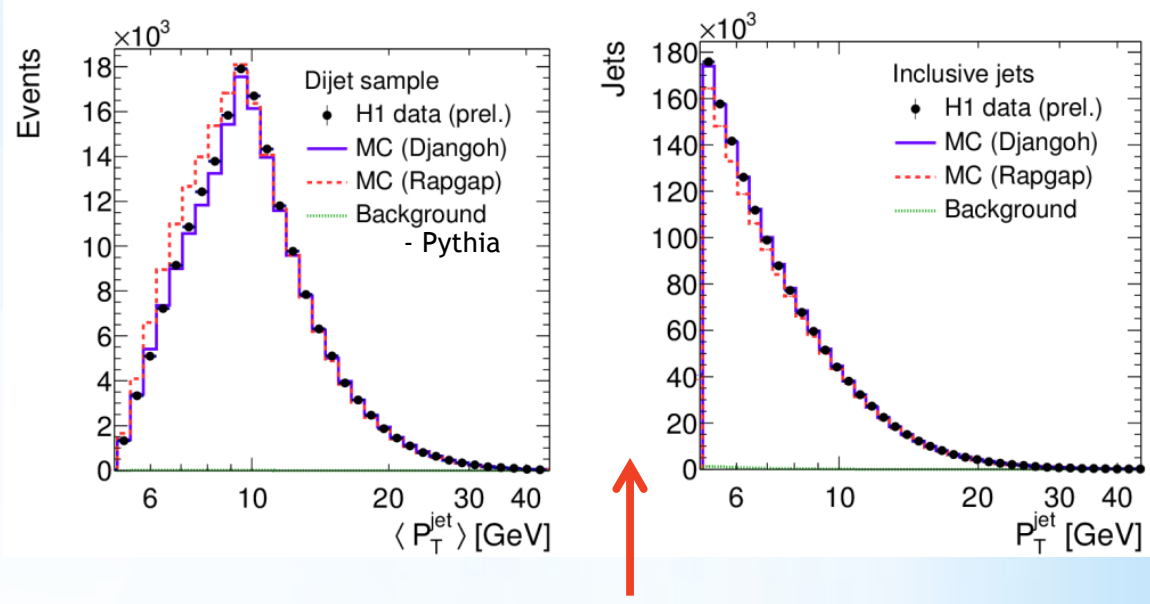

MCs weighted to achieve a better description of data

Hadronisation corrections to NLO,NNLO:

- the average of corrections from RAPGAP and DJANGOH

- multiplicative factors, typically 0.86-0.97 for trijet at low $<\mathrm{P}_{\mathrm{T}}>$ up to 0.73

- uncertainty defined as difference between (RAPGAP - DJANGOH)/2

Correction applied to data: Data are corrected for QED radiative effects 


\section{Inclusive jets:}

-count each jet with $\mathrm{P}_{\mathrm{T}}$ jet $>4.5 \mathrm{GeV}$ in $\mathrm{NC}$ DIS event

Systematic uncertainties dominated by jet and cluster energy, scale and model uncertainty

\section{NNLO predictions:}

-the description of the data improved in comparison with NLO predictions (mainly for low $\mathrm{P}_{\mathrm{T}}$ jet and low $\mathrm{Q}^{2}$ )

\section{aNNLO predictions:}

-the description of the data improved at higher values of $\mathrm{P}_{\mathrm{T}}$ jet as compared to NLO predictions

$$
\begin{gathered}
\sigma_{i}=\sigma_{i}^{j e t} / \sigma_{i Q 2} \\
\uparrow
\end{gathered}
$$

Inclusive jets - normalised to NC DIS x-sections Ratio $\sigma_{\mathrm{i}} / \sigma_{\text {iNLO }}$
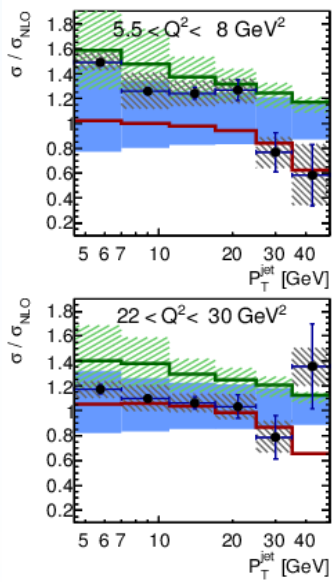

Norm. inclusive jet

† H1 HERA-II (prel.)

$\uparrow$ H1 HERA-II

Systematic uncertainty

NLO $\otimes$ hadr. corr.

New predictions

W/. NNLLO $\otimes$ hadr. corr.

—
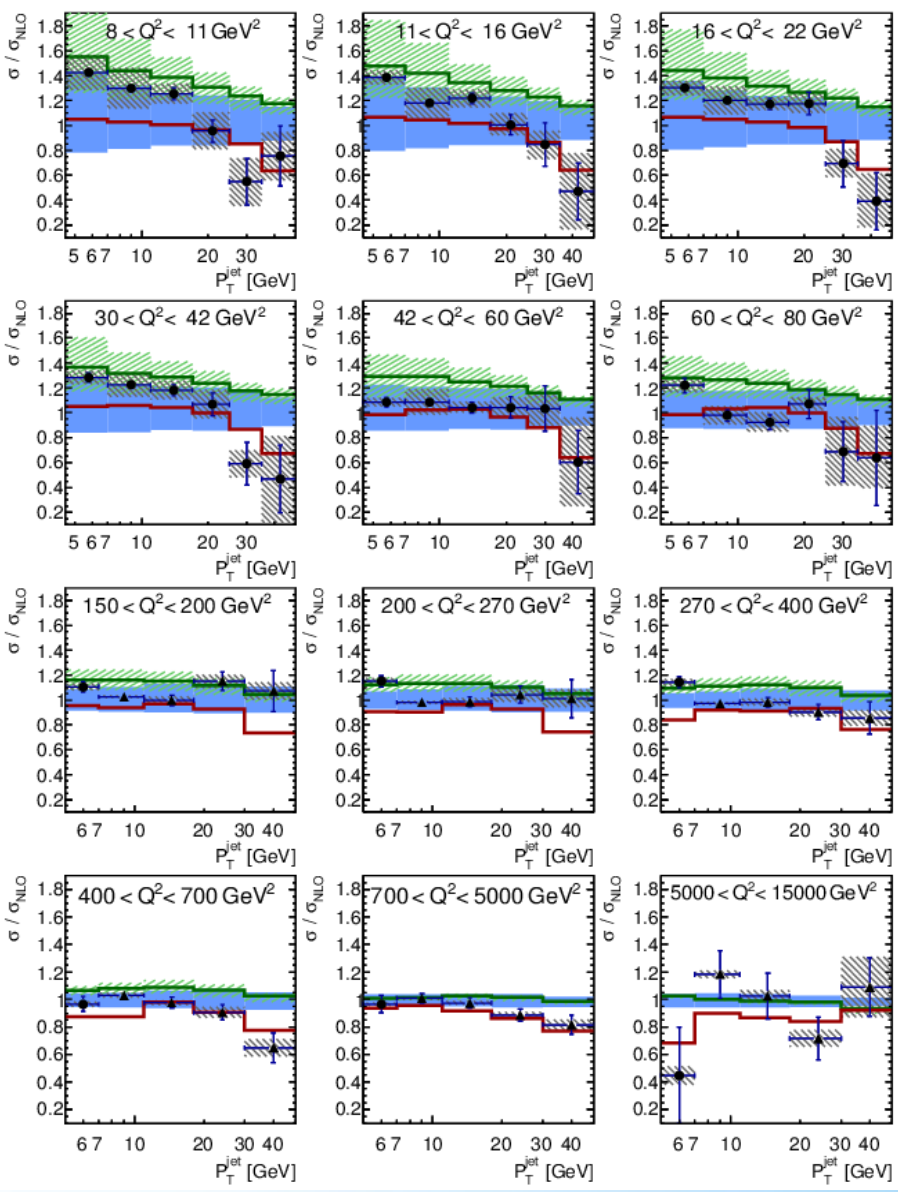


\section{Dijets - NLO and NNLO}

\section{NNLO predictions:}

-the description of the data improved in comparison with NLO predictions (mainly for low $\mathrm{P}_{\mathrm{T}}$ jet and low $\mathrm{Q}^{2}$ )

-significant improvement of the shape description

-slightly higher in normalisation (partially due to the normalisation to NC DIS cross sections)
Dijets - normalised to NC DIS x-sections

Ratio $\sigma / \sigma_{\mathrm{NLO}}$

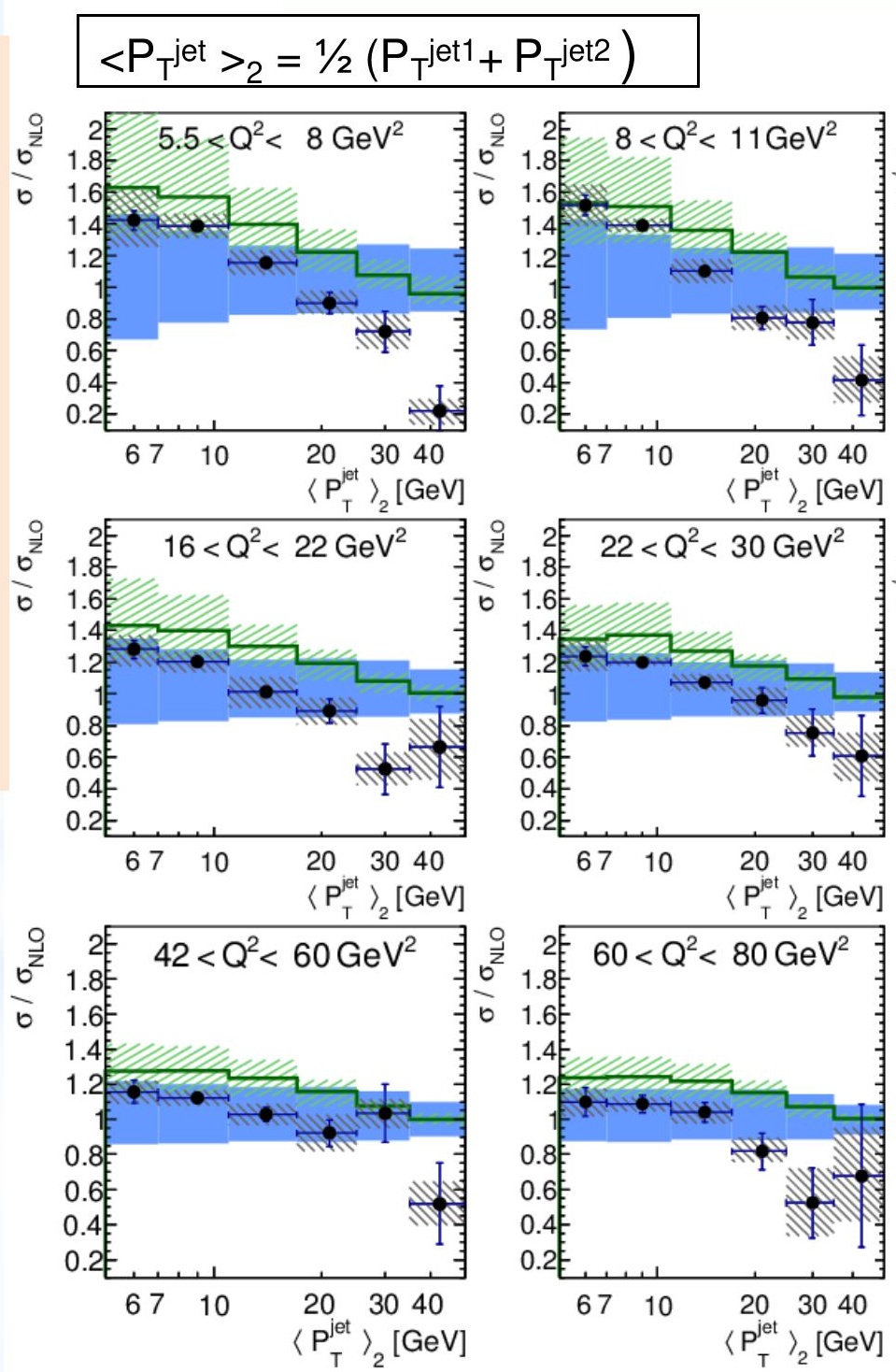

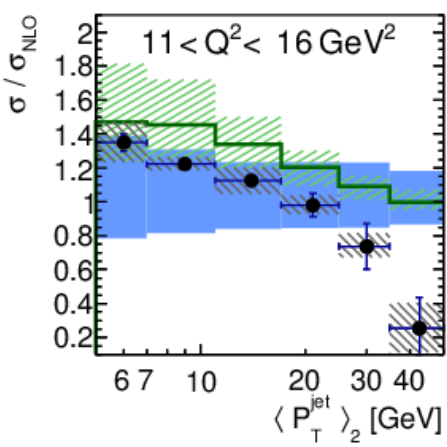

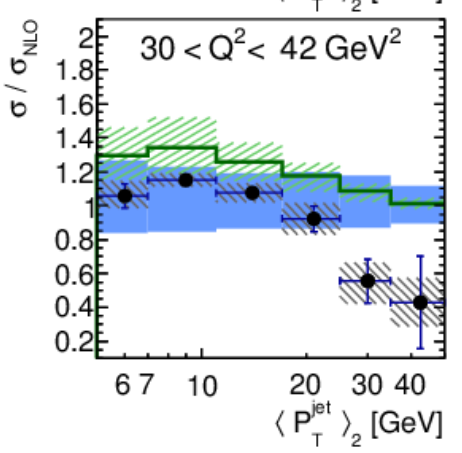

Normalised dijet

† H1 HERA-II (prel.)

Systematic uncertainty

NLO $\otimes$ hadr. corr.

New predictions

NNLO $\otimes$ hadr. corr.

Whys. Rev. Lett. 117 (2016) 042001 
Trijets - normalised to NC DIS x-sections Ratio $\sigma / \sigma_{\text {NLO }}$

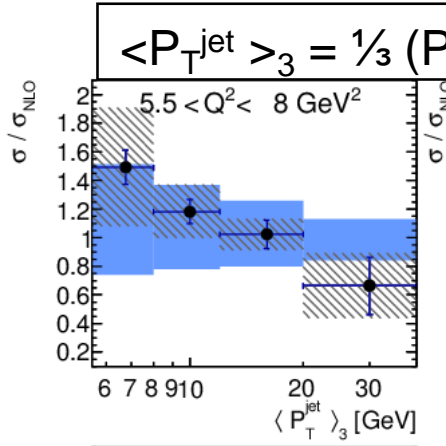

$\left(\mathrm{P}_{\mathrm{T}}^{\mathrm{jet} 1}+\mathrm{P}_{\mathrm{T}}^{\mathrm{jet} 2}+\mathrm{P}_{\mathrm{T}}^{\mathrm{jet} 3}\right)$
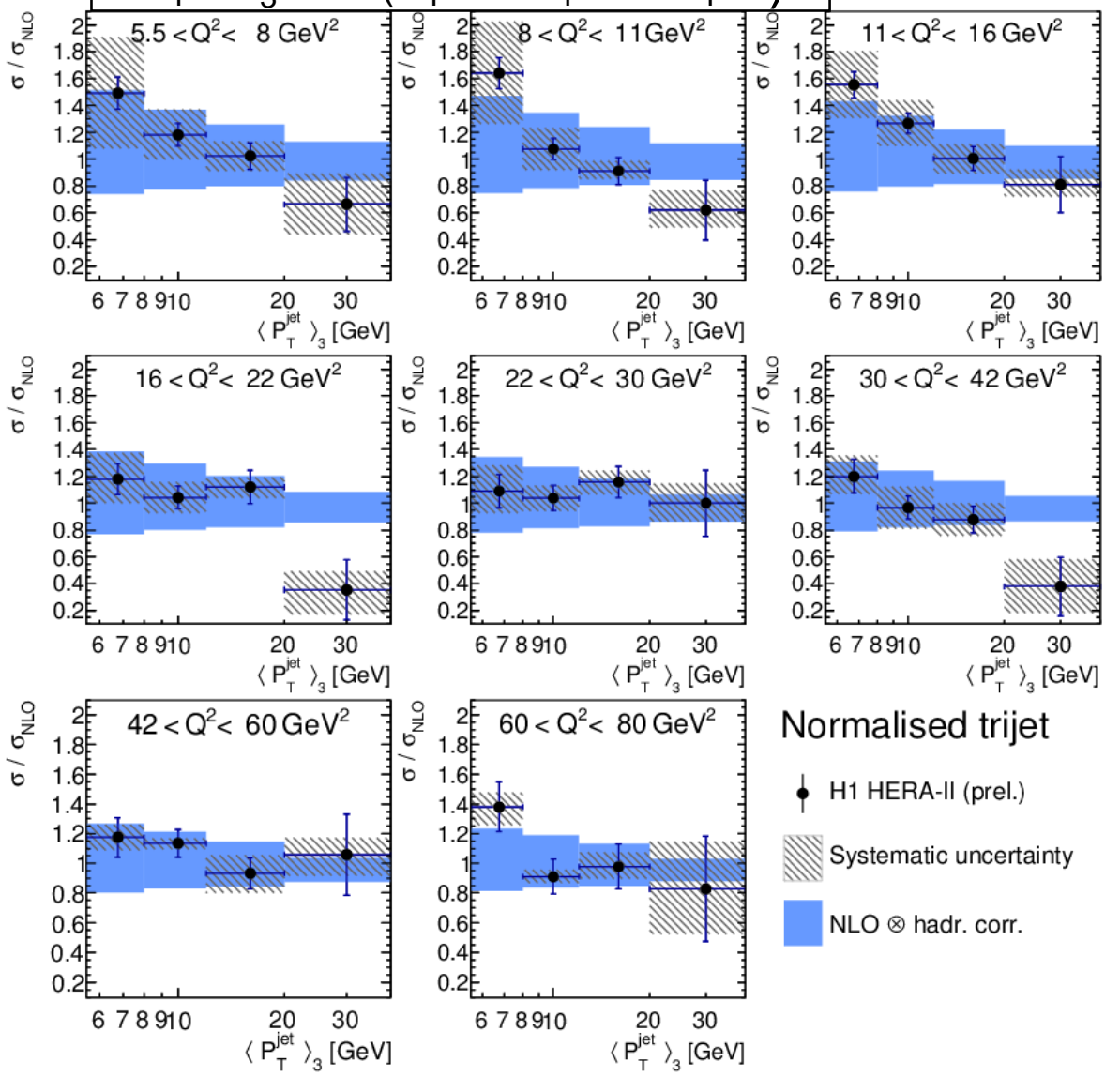

Normalised trijet

† H1 HERA-II (prel.)

Systematic uncertainty

$\mathrm{NLO} \otimes$ hadr. corr.

\section{-Data well described by NLO within} large experimental and theoretical uncertainties $\alpha_{s}\left(m_{z}\right)$ from normalised low- $Q^{2}$ multijets \& NLO

- probe running of $\alpha_{s}(\mu)$ in range $6<\mu<30 \mathrm{GeV}$

- Normalised low- $\mathrm{Q}^{2}$ and high- $\mathrm{Q}^{2}$ multijets experimental precision about $0.4 \%$

The deviation in low $\mu_{\mathrm{r}}$ in accordance with observed enhancement of NNLO vs NLO and data jets!

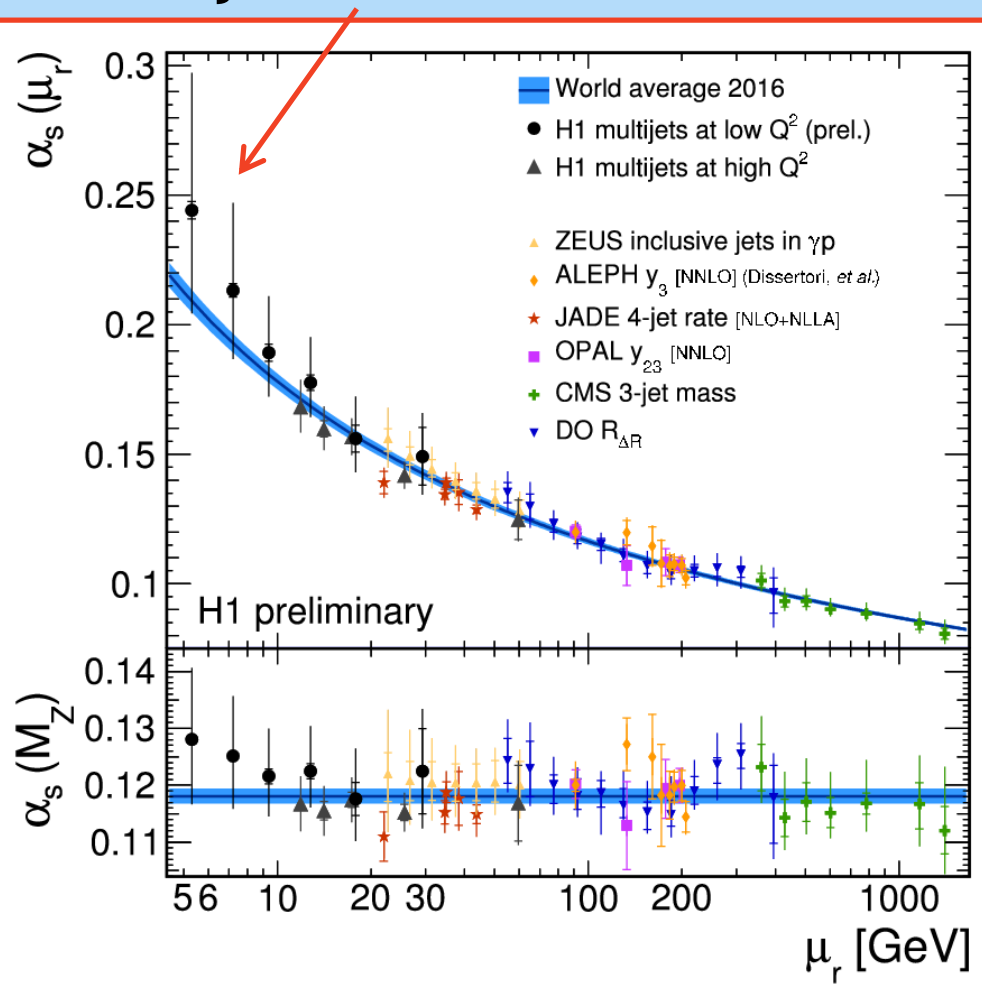




\section{Search for Instantons}

Confirmation of non-perturbative QCD of SM?

Signature:

- Hard current jet

- Instanton band - high multiplicity

- Isotropy in I rest frame

- Parton $(u, d, s)$ democracy

Theory: for HERA $\sigma \sim 10-100 \mathrm{pb}$

A.Ringwald,F.Schrempp a.o.

HERA I data - not observed by H1 and ZEUS, upper limits compatible with theory

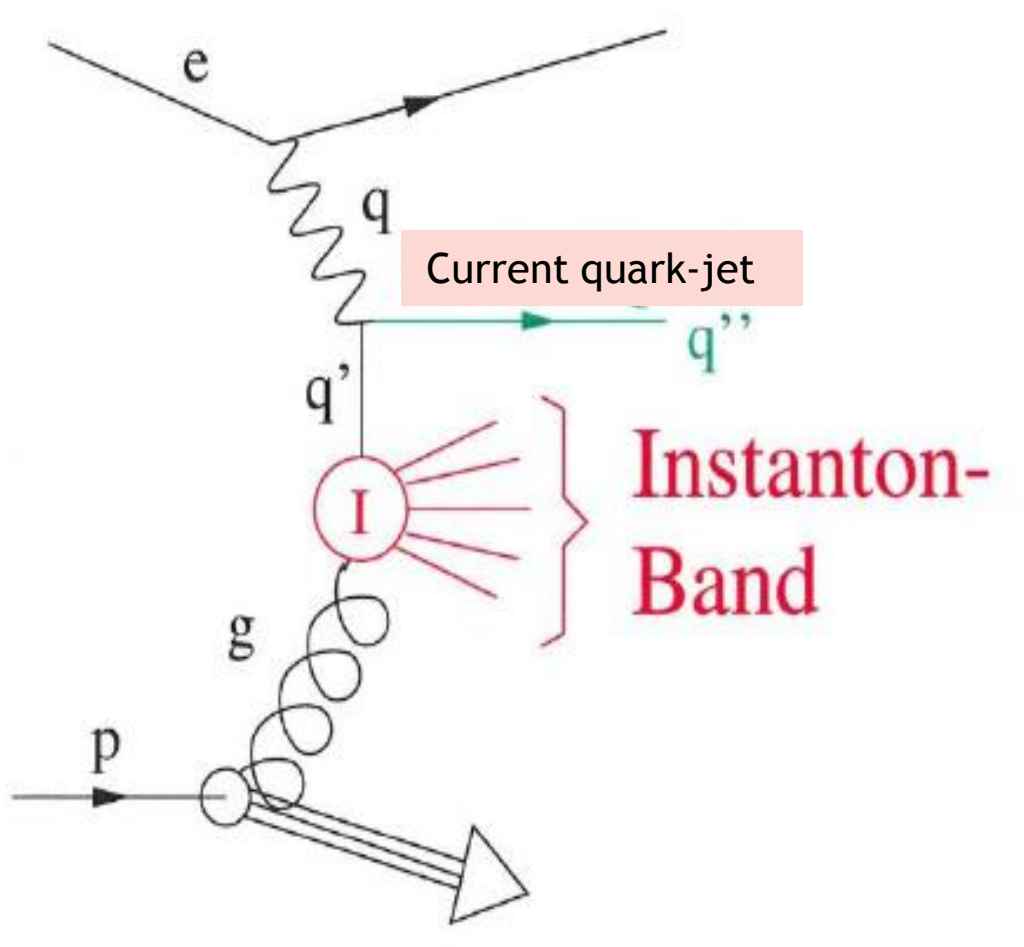

HERA II : data sample $~ 351 \mathrm{pb}^{-1}$

Predicted $\mathrm{x}$-section in studied phase space:

$$
\begin{gathered}
150<\mathrm{Q}^{2}<15000 \mathrm{GeV}^{2}, 0.2<\mathrm{y}<0.7 \\
\mathrm{Q}^{\prime} 2=-\mathrm{q}^{\prime} 2>109 \mathrm{GeV}^{2}, \mathrm{X}^{\prime}=\mathrm{Q}^{\prime} 2 /\left(2 \mathrm{~g} \cdot q^{\prime}\right)>0.35 \\
\sigma(\mathrm{I})=10 \pm 3 \mathrm{pb}
\end{gathered}
$$

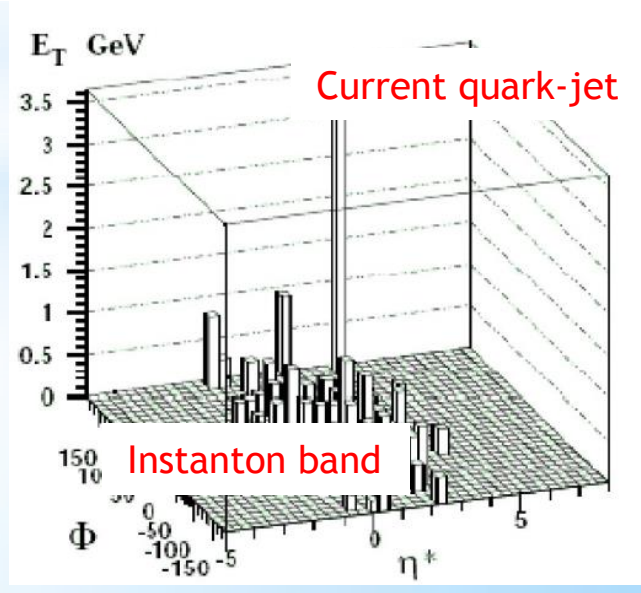




\section{Search for Instantons}

Background needs to be reduced by at least 2 orders of magnitude MultiVariate analysis

$\rightarrow 5$ variables as input to MVA (number of particles, transverse energy of the band, sphericity, Fox-Wolfram moments) - PDERS method (ROOT TMVA package)

- training with RAPGAP/DJANGOH as background and QCDINS as signal MC

- good signal/background separation

- reasonable background description

Signal region

$\mathrm{D}>0.86$

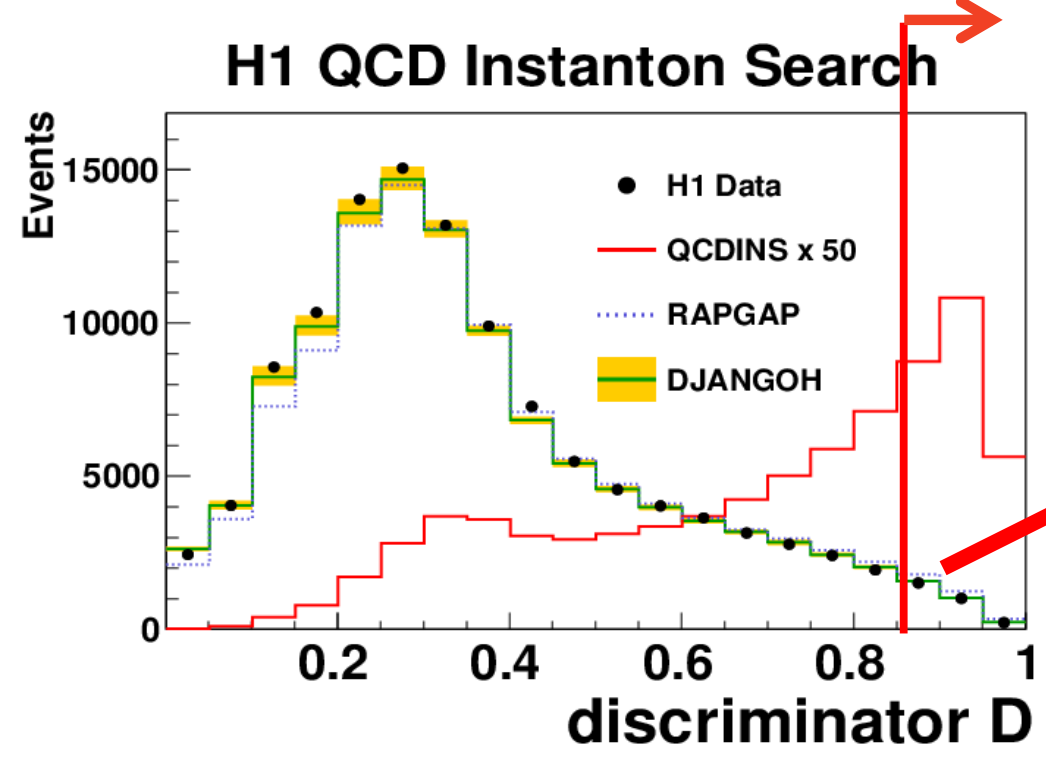

H1 QCD Instanton Search

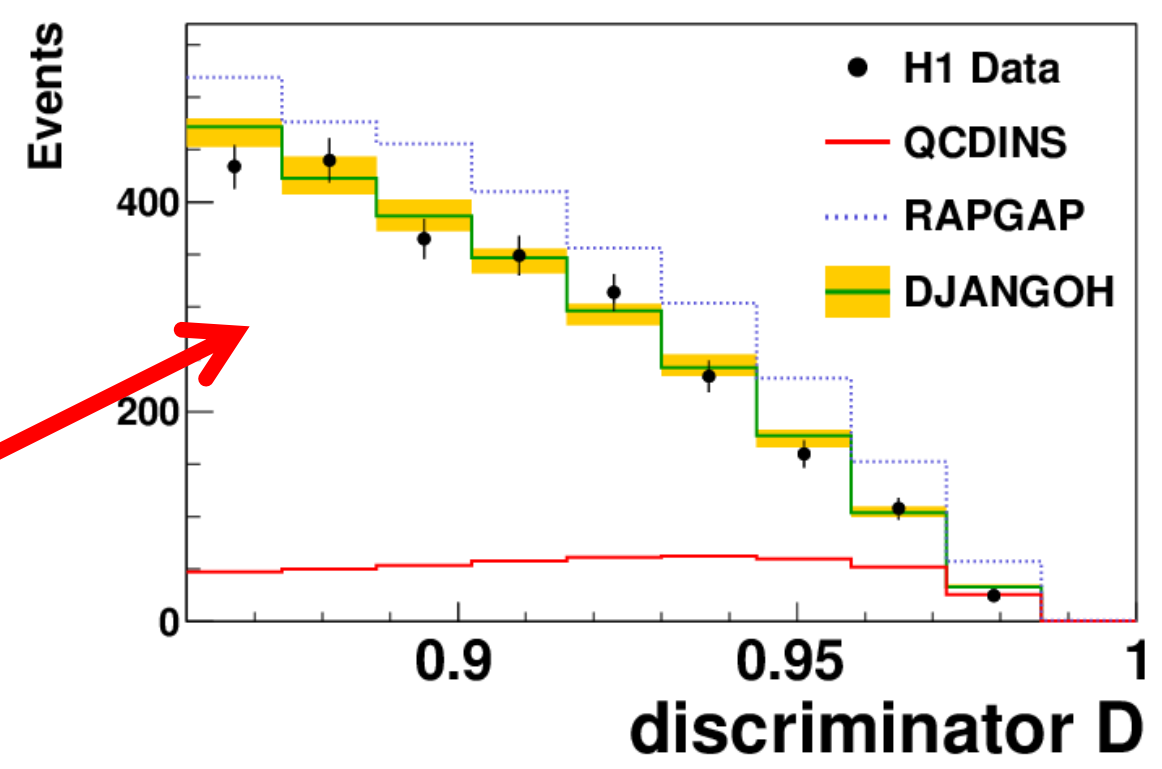

Good description by DJANGOH in signal-background region 


\section{Search for Instantons}

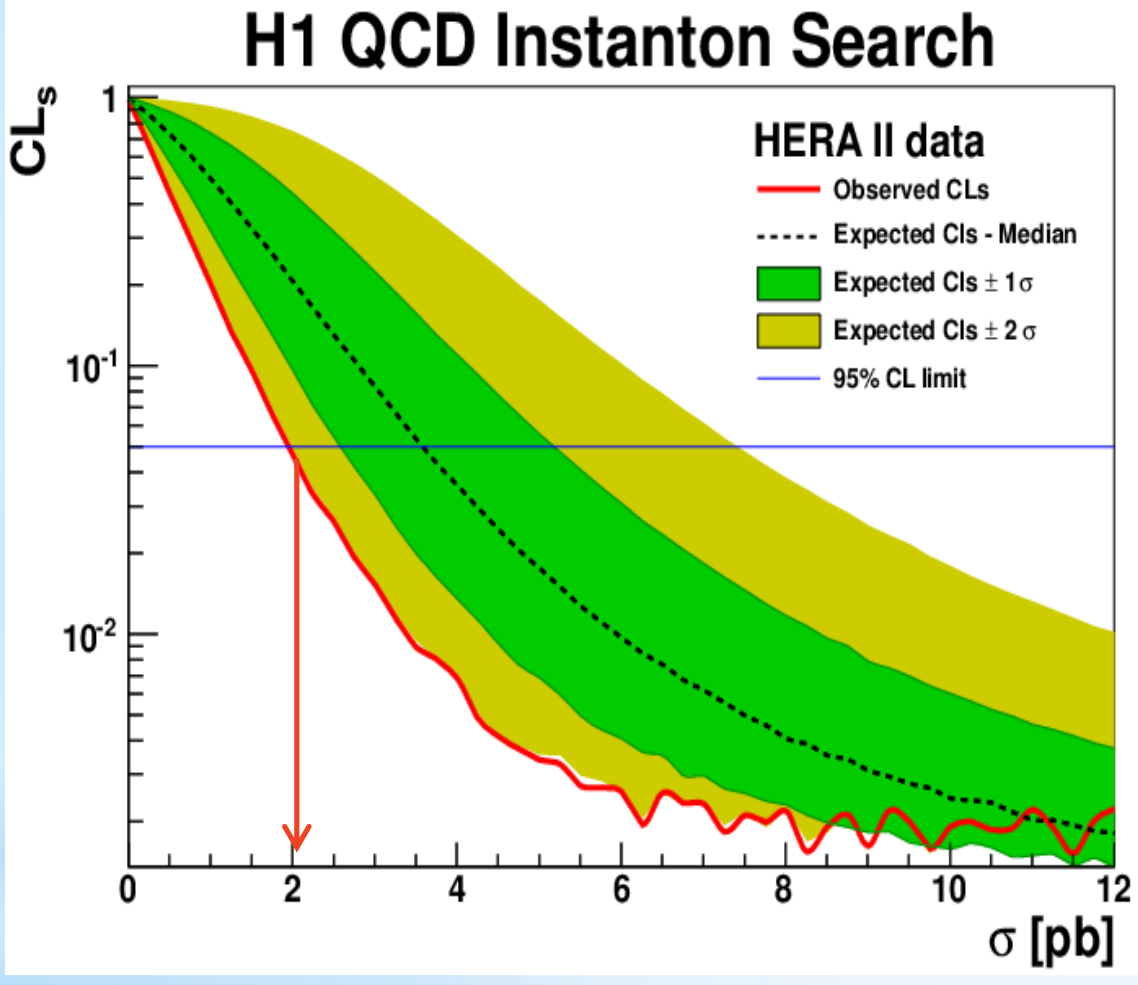

- CLs method

- Using full range discriminator

- Background is DJANGOH

Observed Upper Limit:

$2 \mathrm{pb}$ at $95 \% \mathrm{CL}$

Published in Eur.Phys.J.C76 (2016) 7 


\section{Pentaquarks}

In 2015 LHCb -: possible discovery of two pentaquark states at 4.38 and $4.45 \mathrm{GeV}$ corresponding to uudc $\bar{c}$.

Pentaquarks are topical again!

Early 2000`s reported exotic objects consisting with 5 quarks.

ZEUS: evidence for a peak in $\mathrm{pK}_{\mathrm{s}}^{0}\left(\mathrm{pK}_{\mathrm{s}}^{0}\right)$ corresponding to uudds state at $1.52 \mathrm{GeV}$ (HERA I), $\Theta$ state? Phys.Lett.B 591 (2004) 7

H1 - No such a signal seen, Phys.Lett B 639 (2006) 202

$\mathrm{H} 1$ analysis HERA I - peak in D*p spectrum at $3.1 \mathrm{GeV}$ - uudd $\bar{c}$ (2004)

Not confirmed by ZEUS and with HERA II data! (2008)

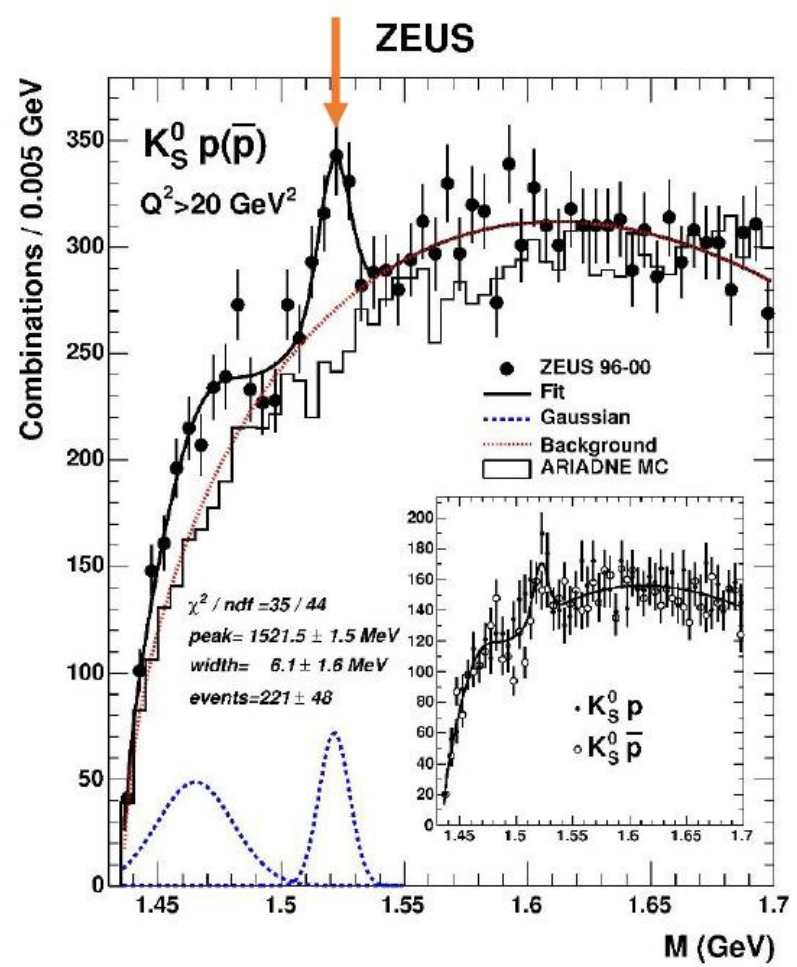




\section{Pentaquarks}

Now HERA II, 3x larger luminosity

The $\mathrm{pK}_{\mathrm{S}}{ }_{\mathrm{S}}$ invariant-mass distribution
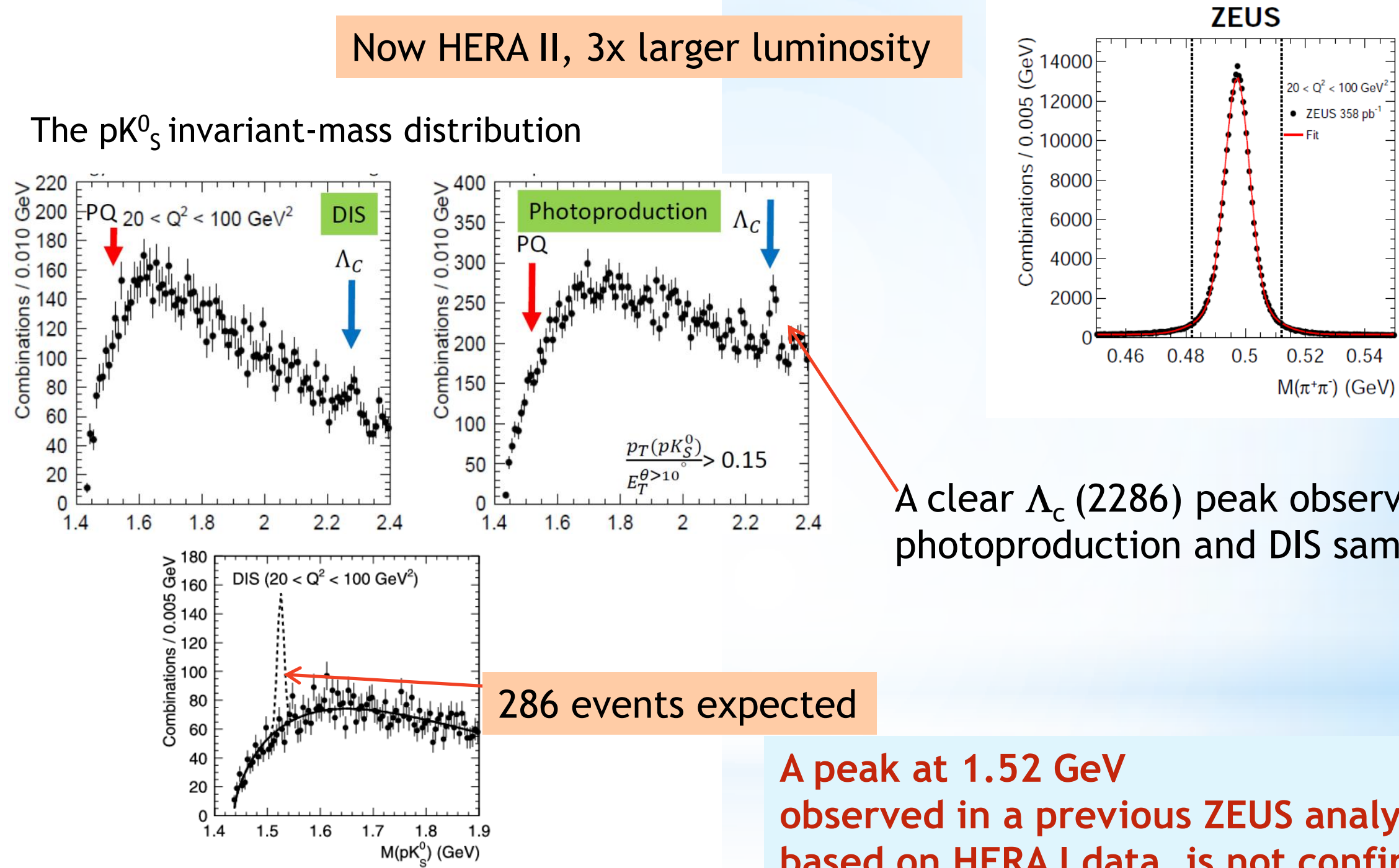

286 events expected

A clear $\Lambda_{c}$ (2286) peak observed in photoproduction and DIS sample

\section{A peak at $1.52 \mathrm{GeV}$}

observed in a previous ZEUS analysis, based on HERA I data, is not confirmed. Published in Phys.Lett. B759 (2016), 446

The dashed line represents the signal corresponding to the ZEUS HERA I result 


\section{Prompt photons in DIS}

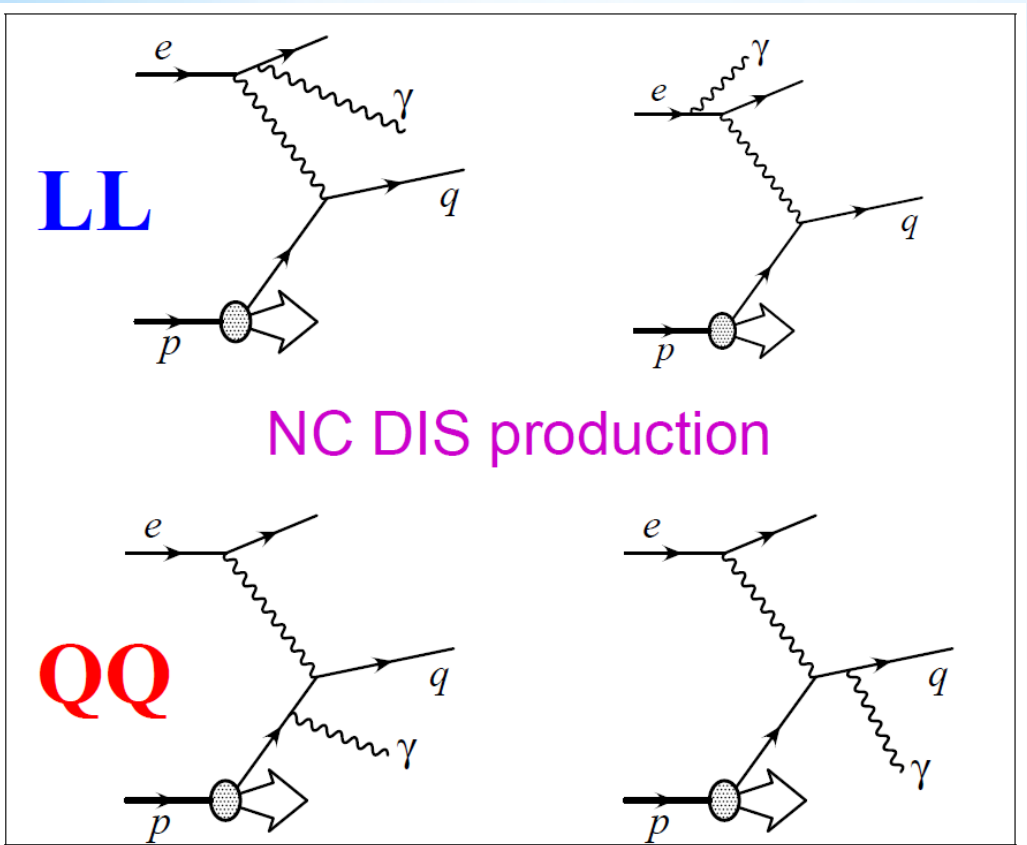

LL-photons are emitted from incoming or outgoing lepton

QQ- photons are emitted from a quark as a part of hard process

Test of QCD, unaffected by hadronisation

- Free of hadronisation corr. for photon $>>$ direct link to parton level

- Sensitivity to parton and photon PDFs

- Important SM background to possible New physics

- Low statistics as compared to jets

- Difficult background from $\pi / \eta /$ decays $>$ systematics $~ 5-10 \%$ 


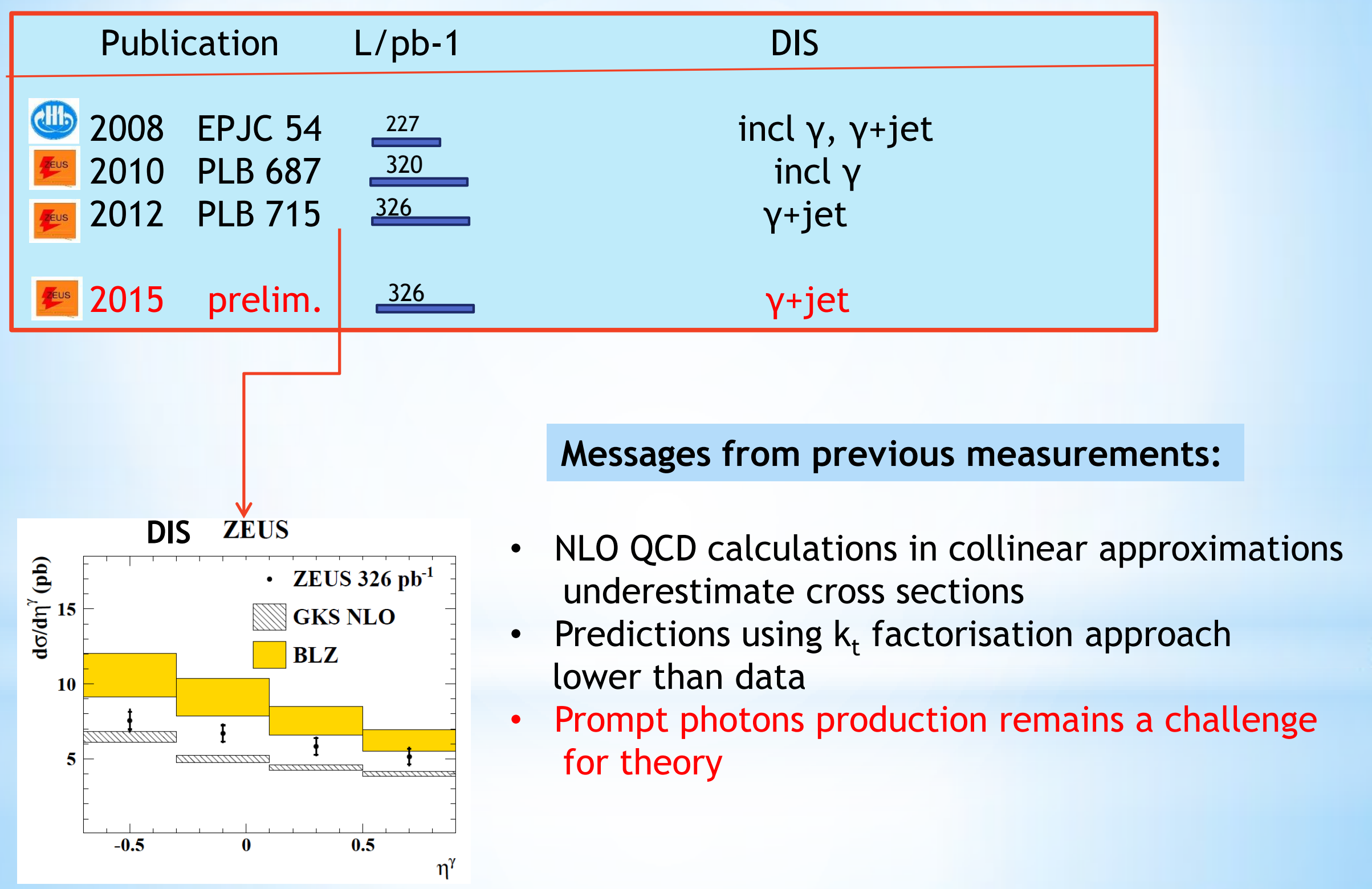




\section{Models used for comparison}

Signal: QQ photons - MC PYTHIA, DIS events with additional radiation from the quark line

LL photons - MC HERACLES \& DJANGOH, higher QCD effects included using color-dipole model as implemented in ARIADNE

Background: Photonic decays of neutral mesons produced in DIS - DJANGOH

\section{Theoretical calculations (BLZ):}

$k_{t}$ - factorization QCD approach

Baranov, Lipatov and Zotov, Phys. Rev. D 81 (2010) 094034

Photon radiation from the quarks as well as from the lepton is taken into account 


\section{Extraction of the photon signal}

ZEUS preliminary 15-001

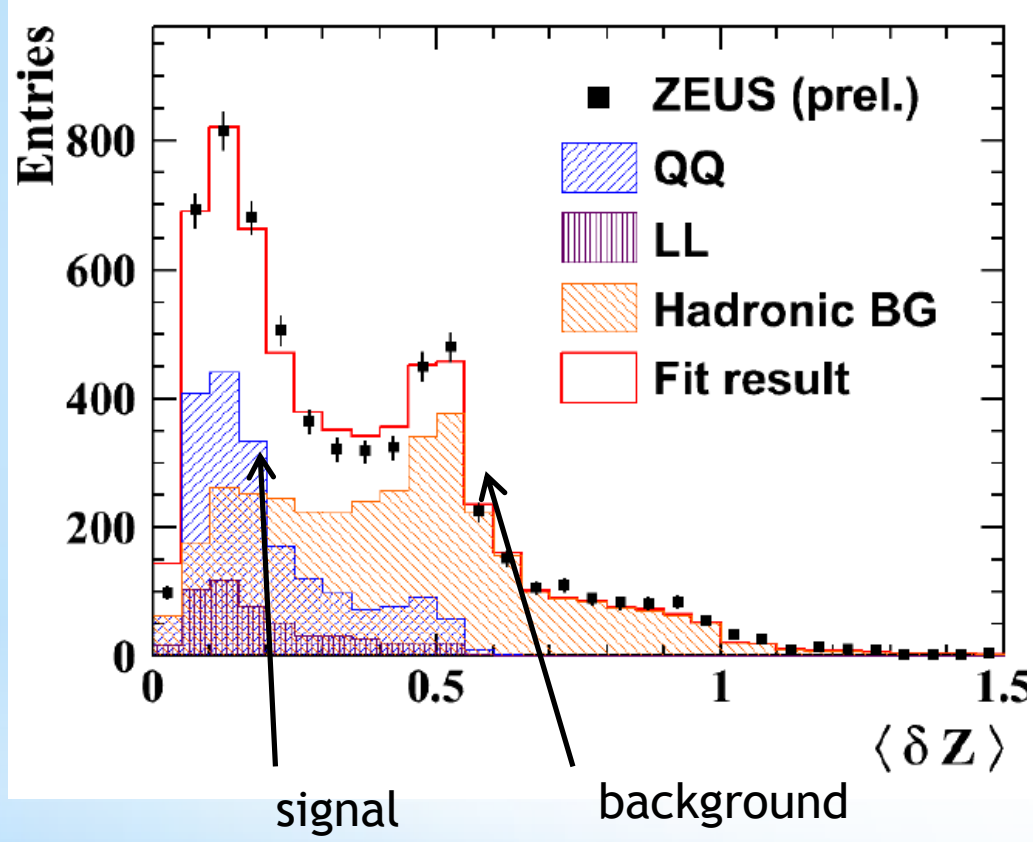

Method to distinguish the signal from hadronic background based on $M C$ fit of $\delta Z$ distribution

Energy-weighted mean width of the electromagnetic shower(cluster) in calorimeter relative to its centroid:

$Z_{i,}\left(Z_{\text {cluster }}\right) Z$ position of the $i$-th cell (centroid of the electromag. cluster), $I_{\text {cell }}$ - width of the cell ,

$\langle\delta Z\rangle=\frac{\sum_{i}\left|z_{i}-z_{\text {cluster }}\right| \cdot E_{i}}{l_{\text {cell }} \sum E_{i}} \quad E_{\mathrm{i}}$ energy recorded in the cell

In each bin of each measured physical quantity, photon signal + hadronic background is fitted

This fit allows to separate statisticaly prompt photon signal (left peak) from background dominated by photons from $\pi^{0}$ decay (right peak)

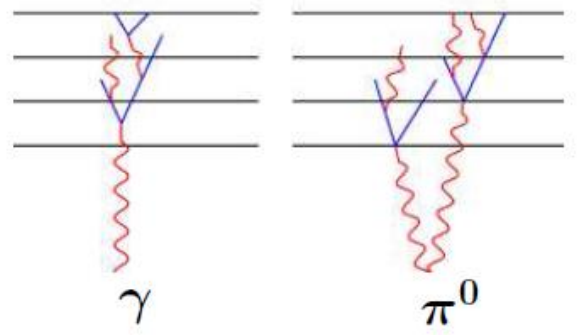




\section{Cross sections compared to weigthed LO MC}

- $x_{\gamma}=\frac{\sum_{j e t, \gamma}\left(E-p_{z}\right)}{2 y_{J B} E_{e}} \cdot \Delta \eta=\eta_{j e t}-\eta_{\gamma}$

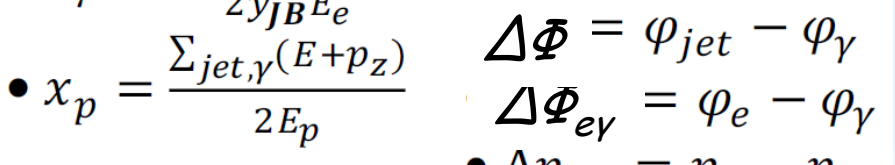

- $\Delta \eta_{e, \gamma}=\eta_{e}-\eta_{\gamma}$
Cross sections compared to

$\mathrm{LL}($ DJANGOHH) + QQ(PYTHIA $) * 1.6$

Shapes are fairly decribed

\section{ZEUS preliminary $15-001$}
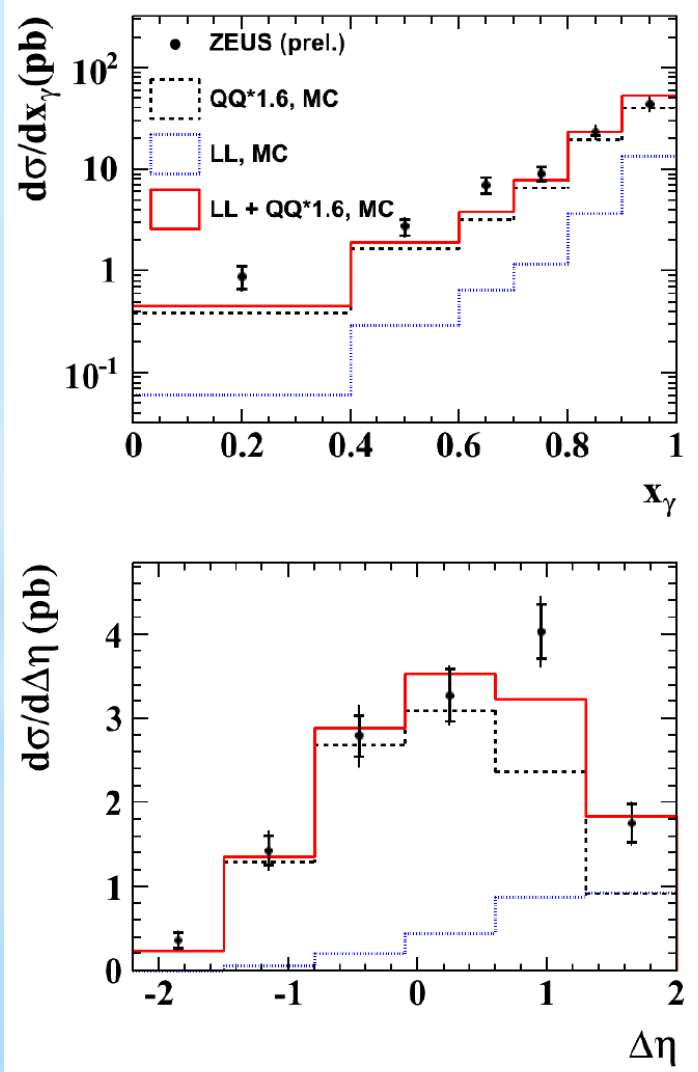
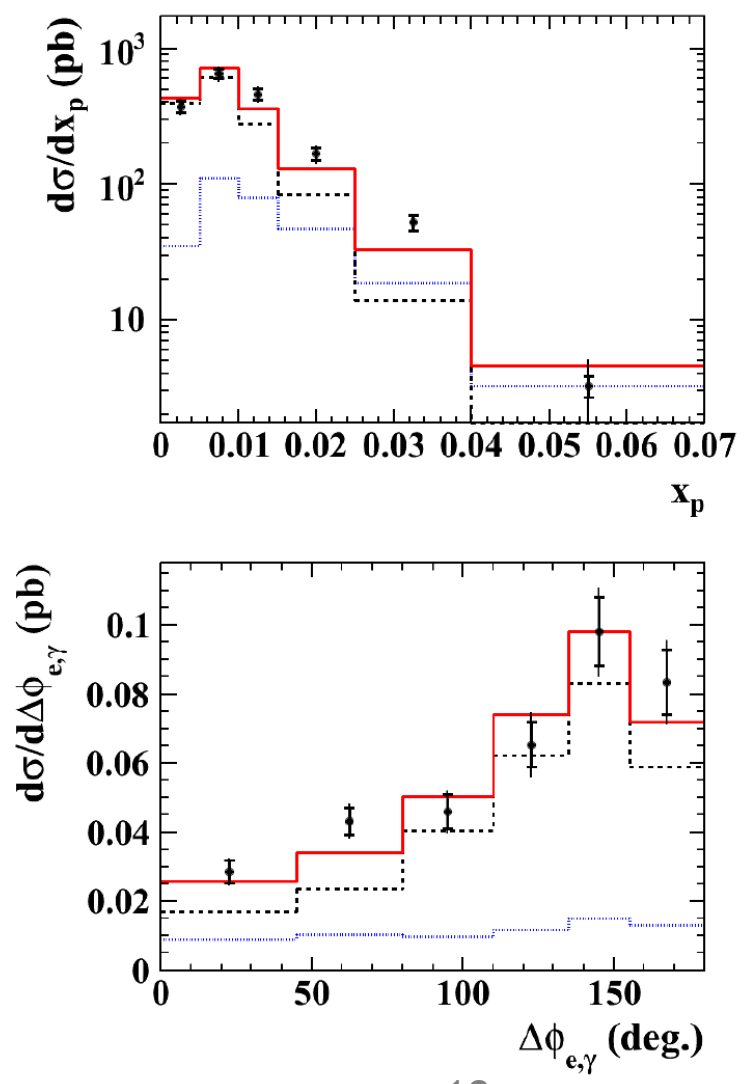

18
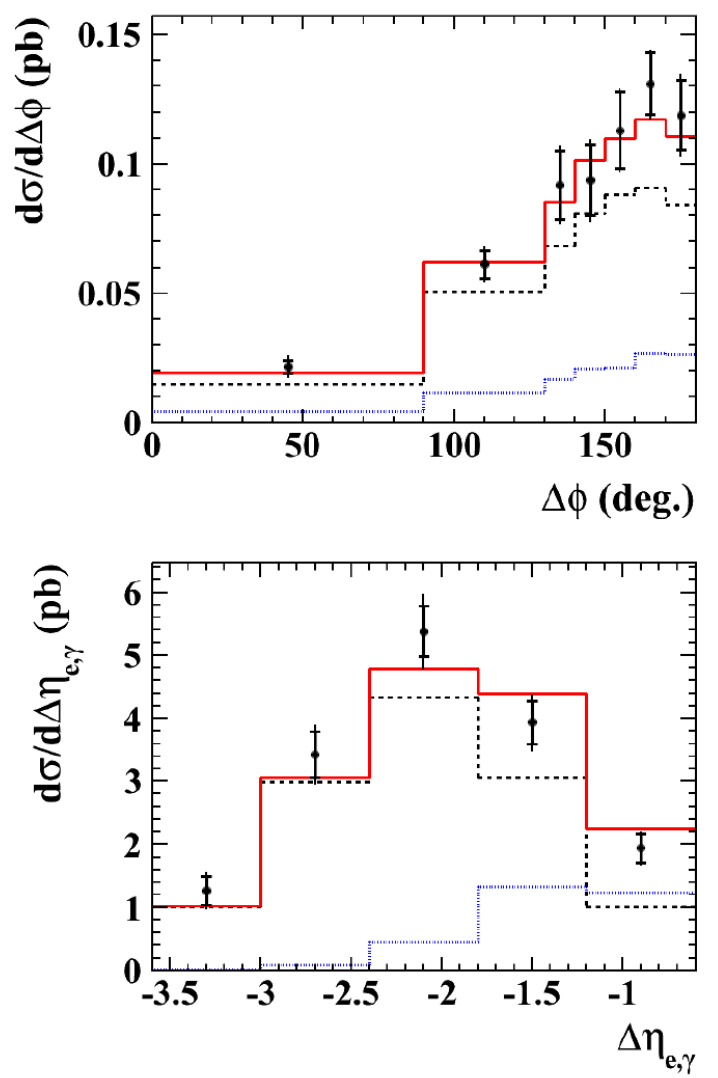


\section{Cross sections compared to $\mathrm{k}_{\mathrm{T}}$ factorisation model}

\section{ZEUS preliminary 15-001}
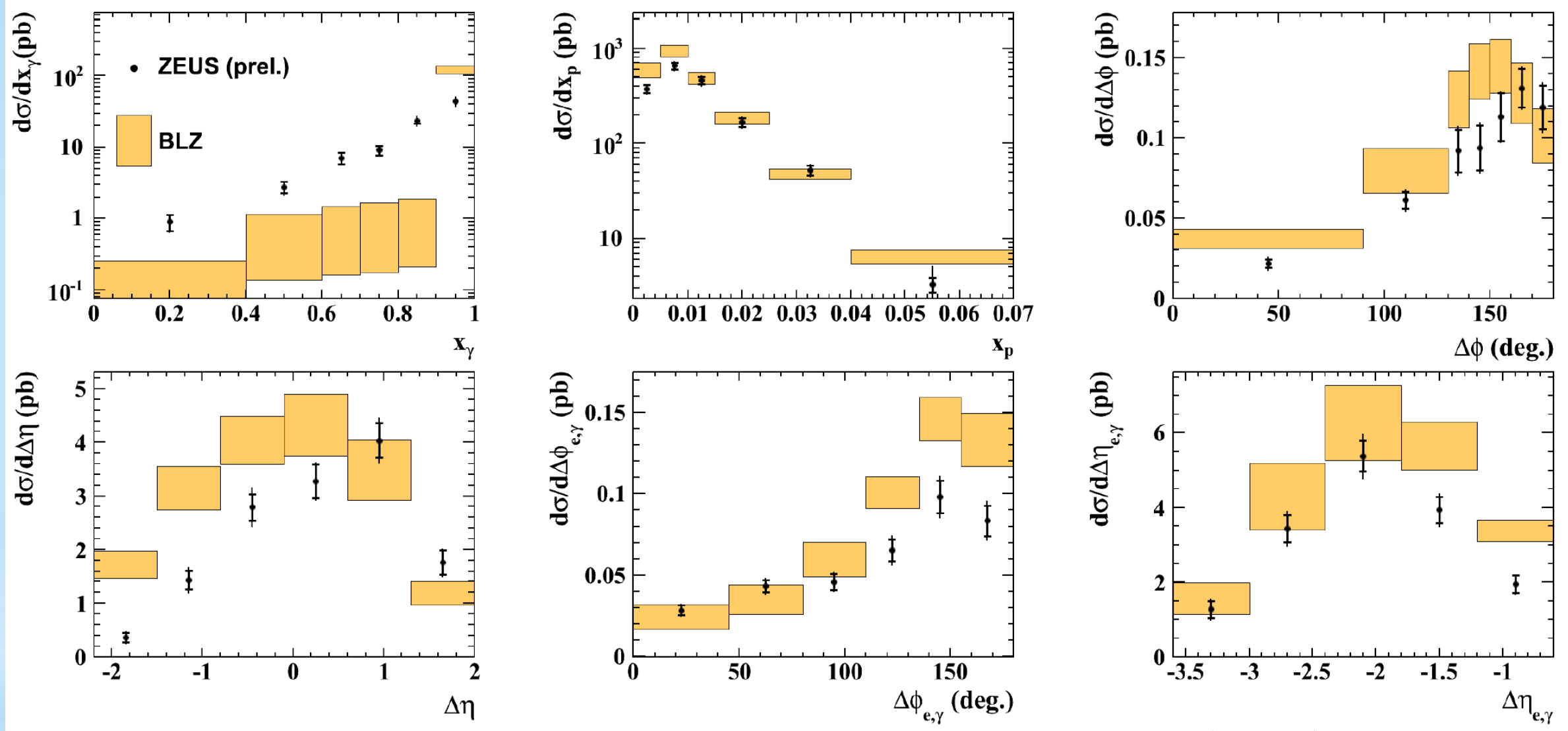

Theoretical calculations, $k_{t}$ - factorization $Q C D$ approach (BLZ)

Baranov, Lipatov and Zotov, Phys. Rev. D 81 (2010) 094034

Photon radiation from the quarks as well as from the lepton is taken into account

BLZ calculations describe shapes of data distributions not so well (mainly $x_{\gamma}$ and $\eta$ ) 


\section{Conclusions}

- New double-differential inclusive jet, dijet and trijet cross sections in DIS obtained,

(iii) inclusive and dijet data compared for the first time with NNLO predictions.

NNLO predictions improve the description of the normalised inclusive and dijet experimental double-differential $\mathbf{x}$-sections compared to NLO.

- Instantos at HERA not found, upper limit $2 \mathrm{pb}$ at $95 \% \mathrm{CL}$, predicted cross section (1iii) $10 \mathrm{pb}$ excluded. Exclusion limits improved by an order of magnitude.

- A resonance in the $\mathrm{pK}_{\mathrm{s}}^{0}\left(\mathrm{pK}_{\mathrm{s}}^{0}\right)$ (pentaquark) at $1.52 \mathrm{GeV}$ from previous ZEUS measurement not confirmed, limits for production cross section established.

- Prompt photons in DIS measured. The predictions for the sum of the expected LL contributions (DJANGOH) and QQ contributions (PYTHIA) rescaled by factor 1.6 $\rightarrow$ good description of the shapes of the kinematic variables. The calculations of BLZ based on $k_{t}$-factorisation method describe the data not so well. 